\title{
Apolipoprotein E4 Frequencies in a Japanese Population with Alzheimer's Disease and Dementia with Lewy Bodies
}

\author{
Seiju Kobayashi ${ }^{1 *}$, Masaru Tateno ${ }^{1}$, Tae Woo Park ${ }^{1}$, Kumiko Utsumi ${ }^{2}$, Hitoshi Sohma ${ }^{3,5}$, Yoichi M. Ito ${ }^{4}$, \\ Yasuo Kokai ${ }^{5}$, Toshikazu Saito ${ }^{1}$
}

1 Department of Neuropsychiatry, Sapporo Medical University School of Medicine, Sapporo, Japan, 2 Department of Psychiatry, Sunagawa City Medical Center, Sunagawa, Japan, 3 Department of Educational Development, Sapporo Medical University Center for Medical Education, Sapporo, Japan, 4 Hokkaido Organization for Translational Research, Hokkaido University Graduate School of Medicine, Sapporo, Japan, 5 Department of Biomedical Engineering, Sapporo Medical University School of Medicine, Sapporo, Japan

\begin{abstract}
Background: The apolipoprotein E (APOE) $\varepsilon 4$ allele has been reported to be a risk factor for Alzheimer's disease (AD) and dementia with Lewy bodies (DLB). Previous neuropathological studies have demonstrated similar frequencies of the APOE $\varepsilon 4$ allele in $A D$ and DLB. However, the few ante-mortem studies on APOE allele frequencies in DLB have shown lower frequencies than post-mortem studies. One reason for this may be inaccuracy of diagnosis. We examined APOE genotypes in subjects with AD, DLB, and a control group using the latest diagnostic criteria and MRI, SPECT, and MIBG myocardial scintigraphy.
\end{abstract}

Methods: The subjects of this study consisted of 145 patients with probable AD, 50 subjects with probable DLB, and a control group. $A D$ subjects were divided into two groups based on age of onset: early onset $A D(E O A D)$ and late onset $A D$ (LOAD). All subjects had characteristic features on MRI, SPECT, and/or myocardial scintigraphy.

Results: The rate of APOE4 carrier status was $18.3 \%$ and the frequency of the $\varepsilon 4$ allele was $9.7 \%$ in controls. The rate of APOE4 carrier status and the frequency of the $\varepsilon 4$ allele were $47 \%$ and $27 \%$ for LOAD, $50 \%$ and $31 \%$ for EOAD, and $42 \%$ and $31 \%$ for DLB, respectively.

Conclusion: The APOE4 genotypes in this study are consistent with previous neuropathological studies suggesting accurate diagnosis of $A D$ and DLB. APOE4 genotypes were similar in AD and DLB, giving further evidence that the $\varepsilon 4$ allele is a risk factor for both disorders.

Citation: Kobayashi S, Tateno M, Park TW, Utsumi K, Sohma H, et al. (2011) Apolipoprotein E4 Frequencies in a Japanese Population with Alzheimer's Disease and Dementia with Lewy Bodies. PLoS ONE 6(4): e18569. doi:10.1371/journal.pone.0018569

Editor: Harm Kampinga, University Medical Center Groningen, University of Groningen, Netherlands

Received January 3, 2011; Accepted March 4, 2011; Published April 28, 2011

Copyright: ๑ 2011 Kobayashi et al. This is an open-access article distributed under the terms of the Creative Commons Attribution License, which permits unrestricted use, distribution, and reproduction in any medium, provided the original author and source are credited.

Funding: This study was supported by the Grant-in-Aid for Young Scientists (B)(MT:22791134) from the Japan Society for the Promotion of Science (JSPS) and Sapporo Biocluster "Bio-S", the Regional Innovation Cluster Program of the Ministry of Education, Sports, Science and Technology (MEXT). JSPS http://www.jsps. go.jp/english/. MEXT http://www.mext.go.jp/english/. The funders had no role in study design, data collection and analysis, decision to publish, or preparation of the manuscript.

Competing Interests: The authors have declared that no competing interests exist.

*E-mail: seij@pastel.ocn.ne.jp

\section{Introduction}

The apolipoprotein $\mathrm{E}$ (APOE) $\varepsilon 4$ allele has been reported to be a risk factor for Alzheimer's disease (AD) [1,2]. APOE is a major component of lipoproteins and plays a role in the metabolism and redistribution of cholesterol [3]. APOE levels increase after brain injury in some neurons and APOE can affect neurite extension. Thus, it is thought to play an important role in the repair and protection of neurons [4]. APOE exists as 3 major alleles $(\varepsilon 2, \varepsilon 3$, and $\varepsilon 4)$ that translate into three isoforms of the protein (APO E2, E3, and E4). Persons being homozygous for the $\varepsilon 4$ allele are more likely to develop the sporadic type of $\mathrm{AD}$ [5].

APOE4 has also been implicated in the development of dementia with Lewy bodies (DLB), thought to be the second most common cause of dementia [6]. Previous neuropathological studies have demonstrated that the frequency of the APOE $\varepsilon 4$ allele in DLB is similar to $\mathrm{AD}[7,8,9,10]$, suggesting a common pathophysiology in the two disorders. However, the few antemortem studies on APOE allele frequencies in DLB have shown lower rates of APOE allele frequencies. One reason may be low accuracy of DLB diagnosis. Previous antemortem studies have not used the most current consensus diagnostic criteria for DLB delineated by the DLB consortium in 2005 [11]. Additionally, radiological testing such as MIBG myocardial scintigraphy was not used for diagnosis.

In this study, we examined APOE genotypes in a population of Japanese subjects diagnosed using relevant radiological testing and the most current consensus diagnostic criteria for DLB and $\mathrm{AD}$. 


\section{Materials and Methods}

\section{Subjects (Table 1)}

The subjects of this study were 279 control subjects from one of our previous studies [12], 145 patients with probable AD according to the NINCDS/ADRDA criteria [13], and 50 subjects with probable DLB according to the latest consensus diagnostic criteria published in 2005 [11]. To obtain non-demented controls, elderly individuals were recruited in Kitahiroshima, Japan and were evaluated by a questionnaire that included an inquiry into past and present illnesses. These population-based non-demented controls consisted of 113 men and 166 women with a mean \pm $\mathrm{SD}$ age at blood draw of $75.6 \pm 8.1$ years. All AD subjects had characteristic neuroimaging features such as atrophy of the parahippocampal gyrus and the parietal lobe on MRI and hypoperfusion in the regions beside the posterior cingulate gyrus on $99 \mathrm{mT}$-ECD brain perfusion SPECT. They were divided into two groups based on the age of onset; early onset AD (EOAD, $<65 \mathrm{y}$ ) and late onset $\mathrm{AD}$ (LOAD, $65 \mathrm{y}$ and higher). Age of onset was determined by interview of patient families. The EOAD group included 16 patients whose mean age was $63.1 \pm 5.0$ years and male:female ratio was 7:9, while the LOAD group consisted of 129 patients whose mean age was $79.1 \pm 4.9$ years and M:F ratio was 44:85. The DLB group consisted of 50 patients whose mean age was 79.1 \pm 4.9 years and M:F ratio was 15:35. All DLB subjects had preserved hippocampal volume on MRI demonstrated by an automated volume measuring program named VARSD [14], occipital hypoperfusion on brain perfusion SPECT revealed by statistical analysis programs [15,16,17,18,19,20,21] and decreased cardiac uptake of 123I- metaiodobenzylguanidine (MIBG) defined as delayed heart-to-mediastinum $(\mathrm{H} / \mathrm{M})$ ratio lower than 1.83 $[17,18,22]$.

The APOE genotyping was performed using the polymerase chain reaction. Informed written consent was obtained from all subjects and their relatives. This study was approved by the institutional ethical committees at Sapporo Medical University.

\section{Method}

APOE genotyping (Figure 1). Using a QIAamp DNA Blood Mini Kit (QIAGEN, Tokyo, Japan), genomic DNA was extracted from the buffy coat after centrifugation of the blood sample $(1 \mathrm{ml})$ according to the manufacture's instructions. DNA genotyping of APOE was performed according to the protocol described by Hixson and Vernier et al [23]. Briefly, leukocyte DNA was amplified by PCR using the oligonucleotide primers Primer 1 (5'TAAGCTTGGCACGGCTGTCGAAGGA-3') and Primer 2 (5'ACAGAATTCGCGCGGGCGTGGTACAC-3') set on common sequence parts of APOE isoforms. The PCR products were digested with Hhal (New England Biolabs. Japan Inc., Tokyo,

Table 1. Subject background.

\begin{tabular}{lllll}
\hline & & & & \\
& Controls & late onset & early onset & \\
& AD & AD & DLB \\
\hline$n$ & 279 & 129 & 16 & 50 \\
Sex (M:F) & $113: 166$ & $44: 85$ & $7: 9$ & $15: 35$ \\
Age (y) & $75.6 \pm 8.1$ & $79.1 \pm 4.9$ & $63.1 \pm 5.0$ & $79.1 \pm 4.9$ \\
Age at onset (y) & & $75.6 \pm 5.2$ & $59.3 \pm 5.9$ & $75.5 \pm 5.0$ \\
MMSE score & & $15.5 \pm 5.5$ & $15.8 \pm 7.2$ & $15.3 \pm 5.4$ \\
Period of education (y) & & $9.1 \pm 2.5$ & $11.2 \pm 1.7$ & $8.6 \pm 3.0$ \\
\hline doi:10.1371/journal.pone.0018569.t001 & & &
\end{tabular}

Japan) and the resulting digestion fragments were separated by electrophoresis on polyacrylamide gels (SuperSepTMDNA 15\% gel(Wako, Tokyo, Japan)). Each genotype of APOE was distinguished by unique combinations of Hhal fragment sizes in all homozygotic and heterozygotic combinations [23]. After determining the APOE genotypes, we investigated the APOE4 carrier status and the frequency of the $\varepsilon 4$ allele in the 279 controls, $145 \mathrm{AD}$, and 50 DLB cases.

Statictical Analysis (Table 2). Differences in APOE carrier status between groups were evaluated by the Cochran-MantelHaenszel Test. It was used to test if there is a relationship between patients (LOAD, EOAD, and DLB) and controls after blocking across a third classification: Wild, Hetero (heterozygous), and Homo (homozygous). That is to say, these comparisons was performed in $2 \times 3$ contingency tables. The frequencies of the $\varepsilon 4$ allele between groups were compared in $2 \times 2$ contingency tables using the Fisher's Exact Test. All analyses were conducted with JMP version 7 (SAS Institute Inc., Cary, NG).

\section{Results}

The results are shown in Table 2.

In the control group, 51 out of 279 subjects were APOE4 carriers $(18.3 \%)$. Three subjects were homozygous for the $\varepsilon 4$ allele $(1.1 \%)$ and 48 subjects were heterozygous for the $\varepsilon 4$ allele $(17.2 \%)$.The total frequency of the $\varepsilon 4$ allele was $9.7 \%$.

In the LOAD group, 61 out of 129 subjects were APOE4 carriers $(47.3 \%)$. Nine subjects were homozygous for the $\varepsilon 4$ allele $(7.0 \%)$ and 52 subjects were heterozygous for the $\varepsilon 4$ allele $(40.3 \%)$. The total frequency of the $\varepsilon 4$ allele was $27.1 \%$.

In the EOAD group, 8 out of 16 subjects were APOE 4 carriers (50\%). Two subjects were homozygous for the $\varepsilon 4$ allele $(12.5 \%)$ and 6 subjects were heterozygous for the $\varepsilon 4$ allele $(37.5 \%)$. The total frequency of the $\varepsilon 4$ allele for this group was $31.3 \%$.

In the DLB group, 21 out of 50 subjects were APOE4 carriers $(42 \%)$. Three subjects were homozygous for the $\varepsilon 4$ allele $(6.0 \%)$ and 18 subjects were heterozygous for the $\varepsilon 4$ allele (36\%). The total frequency of the $\varepsilon 4$ allele was $24 \%$.

APOE carrier status was significantly different between LO $\mathrm{AD}$ and controls $(p<0.0001)$, between EOAD and controls $(p=0.0002)$, and between DLB and controls $(p=0.0003)$. Allele frequencies of APOE $\varepsilon 4$ were significantly higher in LOAD $(p<0.0001), \operatorname{EOAD}(p=0.0011)$, and DLB $(p<0.0002)$ than in controls (Table 2). There were no significant differences in rates of APOE4 carrier status $(p=0.82)$ and the frequencies of the $\varepsilon 4$ allele $(p=0.59)$ between LOAD and DLB. No significant differences in rates of APOE4 carrier status $(p=0.66)$ and the frequencies of the $\varepsilon 4$ allele $(p=0.49)$ were found between EOAD and DLB. Similarly, no significant differences were found in rates of APOE4 carrier status $(p=0.73)$ and the frequencies of the $\varepsilon 4$ allele $(p=0.68)$ between LOAD and EOAD.

\section{Discussion}

This study examined the rate of APOE4 carrier status and the frequency of the $\varepsilon 4$ allele in a group of non-demented subjects, subjects with probable $\mathrm{AD}$ who were separated into early-onset and late-onset groups, and subjects with DLB. A significant difference in the distribution of APOE phenotypes was found between each patient group (LOAD, EOAD, and DLB) and controls. We found no significant difference in rates of APOE4 carrier status and the frequencies of the $\varepsilon 4$ allele between LOAD/ EOAD and DLB subjects. The APOE genotypes in our control subjects are similar to those reported in control subjects from other Japanese studies [24,25]. 
a view showing a frame format of ApoE amino acid arrangement

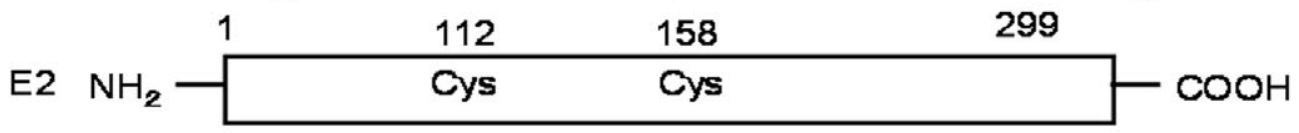

E3 $\mathrm{NH}_{2} \longrightarrow$ Cys Arg $-\mathrm{COOH}$

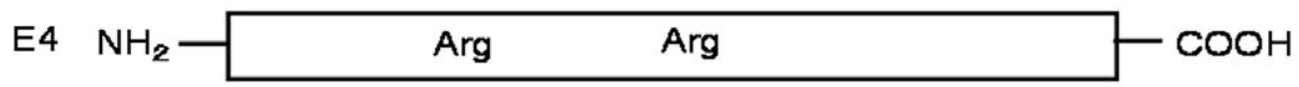

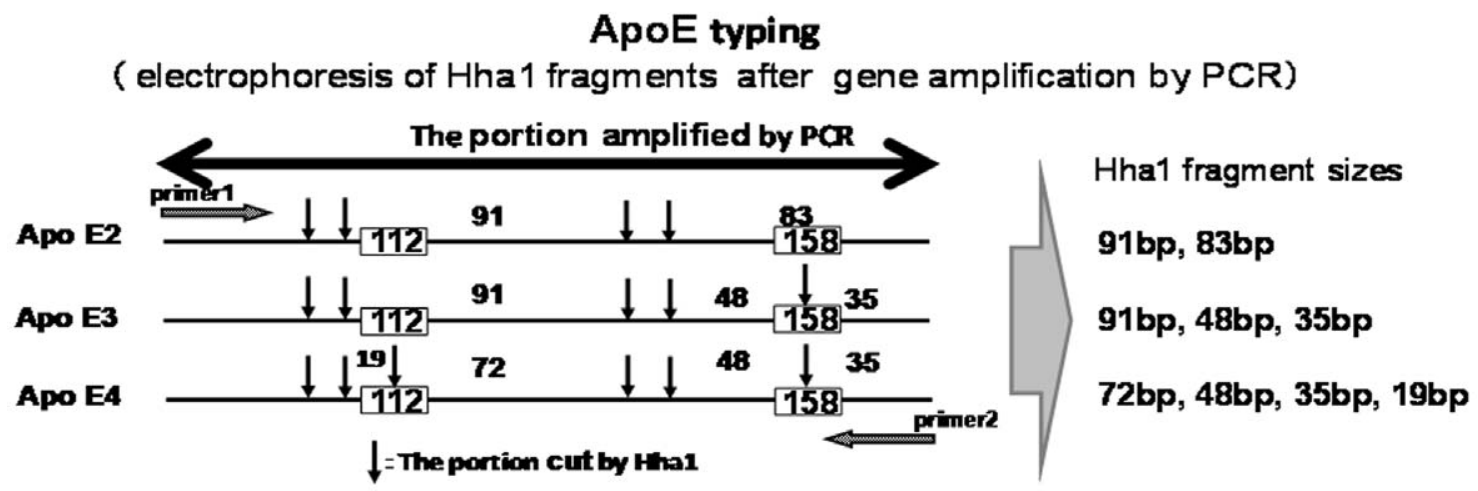

Figure 1. ApoE isoform and Hha1cleavage maps. Each genotype of apoE was distinguished by unique combinations of Hha1 fragments. Cys:Cysteine, Arg:Arginine.

doi:10.1371/journal.pone.0018569.g001

Our study results amongst the AD and DLB patients are similar to previous postmortem studies examining APOE4 carrier status and $\varepsilon 4$ allele frequencies. In postmortem studies examining LOAD, the frequency of APOE4 carrier status ranges from $47 \%$ to $59 \%$, and that of the $\varepsilon 4$ allele ranges from $27 \%$ to $37 \%[7,8]$. Regarding antemortem studies on LOAD, the frequency of APOE4 carrier status is $40 \%$ to $59 \%$, and that of the $\varepsilon 4$ allele is 24 to $31 \%[24,26,27]$. Although there are only a few autopsybased studies that measure APOE4 in $\operatorname{EOAD}[7,10]$, the frequency of APOE4 carrier and $\varepsilon 4$ allele was $52 \%-57 \%$ and around $35 \%$, respectively, in those studies. Previous antemortem studies examining EOAD have been widely divergent. Okuizumi et al. investigated 33 antemortem $\mathrm{AD}$ cases and 11 postmortem cases and reported that the frequency of APOE4 carrier and $\varepsilon 4$ allele is $58 \%$ and $30 \%$, respectively [28]. The frequency of APOE 4 carrier status and the $\varepsilon 4$ allele in EOAD was clinically

Table 2. ApoE4 carrier status and the frequency of the $\varepsilon 4$ allele in Controls, LOAD, EOAD, and DLB.

\begin{tabular}{|c|c|c|c|c|c|c|c|c|}
\hline & $\varepsilon 2 / 2$ & $\varepsilon 2 / 3$ & $\varepsilon 3 / 3$ & $\varepsilon 2 / 4$ & $\varepsilon 3 / 4$ & $\varepsilon 4 / 4$ & $\begin{array}{l}\text { E4 } \\
\text { carrier }\end{array}$ & $\begin{array}{l}\varepsilon 4 \\
\text { allele }\end{array}$ \\
\hline $\begin{array}{l}\text { Contols } \\
N=279\end{array}$ & $0(0)$ & 20(7.1) & 208(74.6) & $2(0.7)$ & $46(16.5)$ & $3(1.1)$ & $18.3 \%$ & $9.7 \%$ \\
\hline LOAD N=129 & $0(0)$ & $5(3.9)$ & $63(48.8)$ & $2(1.5)$ & $50(38.8)$ & $9(7.0)$ & $47.3 \%$ & $27.1 \%$ \\
\hline EOAD N=16 & $0(0)$ & $0(0)$ & $8(50.0)$ & $1(6.2)$ & $5(31.3)$ & $2(12.5)$ & $50.0 \%$ & $31.3 \%$ \\
\hline DLB N $=50$ & $0(0)$ & $5(10.0)$ & $24(48.0)$ & $2(4.0)$ & 16(32.0) & $3(6.0)$ & $42.0 \%$ & $24.0 \%$ \\
\hline
\end{tabular}

reported as $7 \%$ and $4 \%$ by Kawamata et al. [29], and $72 \%$ and $43 \%$ by Dai et al [30], respectively. The widely divergent results suggest that antemortem studies, particularly involving EOAD, might depend strongly on methodological differences. Our results were consistent with previous postmortem studies in both LOAD and EOAD, supporting the accuracy of our diagnoses.

Although there are many clinical [9,30] and autopsy-based studies [31,32] measuring the $\varepsilon 4$ allele in $\mathrm{AD}$, most do not distinguish between EOAD and LOAD. However, both have different genetic backgrounds [33], different rates of progression [34], and different levels of impairment in verbal and visual cognition $[35,36]$. Thus, it is necessary to distinguish EOAD from LOAD for research purposes. The NINCDS/ADRDA criteria support this notion and states that researchers should be aware of two subtypes; "less than $65 \mathrm{y}$ " and "65 y and higher" [13].

With regards to DLB, our results also closely matched postmortem studies examining the frequencies of APOE4 carrier status and the $\varepsilon 4$ allele. Several research groups from various countries have conducted similar investigations with large samples of postmortem brains of DLB patients $[7,9,10]$. The frequency of APOE4 carrier status and the $\varepsilon 4$ allele in these studies ranged from $36 \%$ to $65 \%$ and 19 to $36 \%$, respectively. There have been three previous antemortem studies that have reported the frequency of APOE4 carrier status and the $\varepsilon 4$ allele in DLB patients. Engelborghs et al. [32] reported the frequency of APOE4 carrier status and the $\varepsilon 4$ allele as $28.5 \%$ and $18 \%$, respectively, Carrillo et al. [31] indicated frequencies of $27.6 \%$ and $16.4 \%$, and Lane et al. [37] reported frequencies of $35 \%$ and $18.1 \%$. These frequencies are lower or at the lower end of the range of frequencies determined by autopsy-based studies, as opposed to our results which were more consistent with previous autopsybased studies. 
Accurate antemortem diagnosis of DLB is important. Some patients with DLB may have an accelerated disease progression and approximately $50 \%$ of patients experience life-threatening adverse reactions to antipsychotic medications [38]. Our results suggest that our clinical diagnosis of DLB and AD was quite accurate. To accomplish the highest possible accuracy of antemortem diagnosis of DLB, we performed MRI, brain perfusion SPECT and MIBG myocardial scintigraphy in conjunction with using the latest DLB diagnostic criteria. These diagnostic guidelines [11] acknowledge the importance of the types of neuroimaging findings found in our study such as relative preservation of medial temporal lobe structures on MRI scan, occipital hypoperfusion on brain perfusion SPECT and decreased MIBG uptake on myocardial scintigraphy. We have previously demonstrated the usefulness of these neuroimaging tools for the clinical diagnosis of DLB [18,22]. Notably, all of the previous antemortem studies $[31,32,37]$ on APOE4 in DLB did not use or were conducted before the publication of the current consensus diagnostic criteria for DLB. Our study is the first antemortem study to use the latest diagnostic criteria for DLB. The results of our study suggest that the current criteria used in conjunction with MIBG myocardial scintigraphy, MRI and PET is more accurate in the diagnosis of DLB than previous diagnostic criteria.

There is a growing consensus that APOE allele frequency is influenced by race [39]. This may explain why the frequency of the $\varepsilon 4$ allele in autopsied DLB cases varies widely (19 to $36 \%$ ). To the best of our knowledge, there are no antemortem studies in which APOE4 genotypes are characterized in Asian patients with DLB. A prior meta-analysis found that the APOE $\varepsilon 4$ allele association with $\mathrm{AD}$ was stronger in Japanese subjects than in Caucasian subjects [39]. Our results suggest that the APOE $\varepsilon 4$ allele is more frequent in Japanese patients with DLB.

The role of APOE $\varepsilon 4$ in the pathophysiology of $\mathrm{AD}$ remains controversial. However, it has been reported that the gene dose of APOE $\varepsilon 4$ correlates with the expression of senile plaques $(\mathrm{SP})$ and neurofibrillary tangles (NFT) [2]. SPs are composed of amyloidbeta protein $(A \beta)$ which is deposited outside the neuron. Previous studies have revealed a large quantity of $A \beta$ deposition in the brain of $\mathrm{AD}$ patients with APOE4 [40]. A $\beta$ deposition has been shown in the neuropil and vessel walls of subjects with APOE4 [40]. Notably, the extent of A $\beta$ deposition in APOE4 homozygotes was more severe than APOE4 heterozygotes [40]. On the other hand, NFTs are composed of phosphorylated tau proteins that have accumulated inside the neuron. Animal studies have revealed that transgenic mice expressing human APOE4 had more phosphorylated tau proteins [26].

\section{References}

1. Corder EH, Saunders AM, Strittmatter WJ, Schmechel DE, Gaskell PC, et al. (1993) Gene dose of apolipoprotein E type 4 allele and the risk of Alzheimer's disease in late onset families. Science 261: 921-923.

2. Saunders AM, Strittmatter WJ, Schmechel D, George-Hyslop PH, PericakVance MA, et al. (1993) Association of apolipoprotein E allele epsilon 4 with late-onset familial and sporadic Alzheimer's disease. Neurology 43: 1467-1472.

3. Mahley RW (1988) Apolipoprotein E: cholesterol transport protein with expanding role in cell biology. Science 240: 622-630.

4. Dietschy JM, Turley SD (2004) Thematic review series: brain Lipids. Cholesterol metabolism in the central nervous system during early development and in the mature animal. J Lipid Res 45: 1375-1397.

5. Strittmatter WJ, Roses AD (1996) Apolipoprotein E and Alzheimer's disease. Annu Rev Neurosci 19: 53-77.

6. McKeith I, O'Brien J (1999) Dementia with Lewy bodies. Aust N Z J Psychiatry 33: $800-808$.

7. AkatsuHiroyasu K, YamagataHidehisa, IsojimaDaisuke, KondoIkuko, YamamotoTakayuki, KidaTomoyuki, TakedaMasatoshi, MikiTetsuro, KosakaKenji (2004) Increased incidence of dementia with Lewy bodies in patients carrying the ع4-allele of apolipoprotein E. Psychogeriatrics 4: 8.
Our results demonstrated similar frequencies of APOE4 carrier status and the $\varepsilon 4$ allele in LOAD, EOAD and DLB. Indeed, previous studies have revealed the commonality of pathological findings in DLB and AD. Kosaka reported that many SPs and/or NFTs are present in autopsy cases with DLB. According to his report, DLB can be divided into two forms: a common form and a pure form [41]. In the common form, numerous Lewy bodies can be found with many SPs and/or NFTs in the cerebral cortex, whereas in the pure form, there are few or no senile changes. Kawanishi et al. also reported that SPs, a characteristic feature of $\mathrm{AD}$, were found in the autopsied brains of DLB patients [27]. We speculate that DLB patients with AD pathology tend to have higher frequencies of the $\varepsilon 4$ allele. Perhaps a higher portion of our DLB patient sample have AD pathology, though due to the antemortem nature of our study it is difficult to assess this.Given the similar frequencies of $\varepsilon 4$ allele in $\mathrm{AD}$ and DLB in our study, our results support the idea that there is often overlapping pathology between AD and DLB and that APOE4 plays a role in the pathophysiology of both disorders.

We must recognize some limitations in our study. The number of subjects in our study was small, although we included 50 probable DLB cases, a number higher than previous studies. Also we have not confirmed the diagnosis through neuropathological means. Current guidelines suggest pathological confirmation to give a definite diagnosis of DLB. However, our results suggest that the combination of careful history taking, conscientious examination of clinical features, neuro-psychological cognitive tests, and neuroimaging tools including MRI, brain perfusion SPECT and MIBG myocardial scintigraphy could significantly increase the preciseness of clinical diagnosis.

In conclusion, our study results give further support for APOE4 as a biological marker for the presence of DLB. Our results demonstrated that the rate of APOE carrier status and the frequency of the $\varepsilon 4$ allele in DLB were as high as in AD. Our results were also consistent with postmortem studies suggesting that our diagnosis including brain perfusion SPECT and MIBG myocardial scintigraphy was accurate.

\section{Author Contributions}

Conceived and designed the experiments: SK MT KU. Performed the experiments: SK MT KU. Analyzed the data: SK YMI. Contributed reagents/materials/analysis tools: SK HS YK. Wrote the paper: SK. Revised the manuscript for intellectual content, study supervision and coordination: MT TP KU HS YK TS.
8. Arai H, Higuchi S, Muramatsu T, Iwatsubo T, Sasaki H, et al. (1994) Apolipoprotein $\mathrm{E}$ gene in diffuse Lewy body disease with or without co-existing Alzheimer's disease. Lancet 344: 1307.

9. Galasko D, Saitoh T, Xia Y, Thal LJ, Katzman R, et al. (1994) The apolipoprotein $\mathrm{E}$ allele epsilon 4 is overrepresented in patients with the Lewy body variant of Alzheimer's disease. Neurology 44: 1950-1951.

10. Singleton AB, Wharton A, O'Brien KK, Walker MP, McKeith IG, et al. (2002) Clinical and neuropathological correlates of apolipoprotein $\mathrm{E}$ genotype in dementia with Lewy bodies. Dement Geriatr Cogn Disord 14: 167-175.

11. McKeith IG, Dickson DW, Lowe J, Emre M, O'Brien JT, et al. (2005) Diagnosis and management of dementia with Lewy bodies: third report of the DLB Consortium. Neurology 65: 1863-1872.

12. Yamaguchi M, Kokai Y, Imai S, Utsumi K, Matsumoto K, et al. (2010) Investigation of annexin A5 as a biomarker for Alzheimer's disease using neuronal cell culture and mouse model. J Neurosci Res 88: 2682-2692.

13. McKhann G, Drachman D, Folstein M, Katzman R, Price D, et al. (1984) Clinical diagnosis of Alzheimer's disease: report of the NINCDS-ADRDA Work Group under the auspices of Department of Health and Human Services Task Force on Alzheimer's Disease. Neurology 34: 939-944. 
14. Matsuda $H(2010)$ [Neuroimaging for patients with Alzheimer disease in routine practice]. Brain Nerve 62: 743-755.

15. Kobayashi S, Tateno M, Utsumi K, Takahashi A, Morii H, et al. (2009) Twolayer appearance on brain perfusion SPECT in idiopathic normal pressure hydrocephalus: a qualitative analysis by using easy Z-score imaging system, eZIS. Dement Geriatr Cogn Disord 28: 330-337.

16. Kobayashi S, Tateno M, Utsumi K, Takahashi A, Saitoh M, et al. (2008) Quantitative analysis of brain perfusion SPECT in Alzheimer's disease using a fully automated regional cerebral blood flow quantification software, 3DSRT. J Neurol Sci 264: 27-33.

17. Tateno M, Kobayashi S, Saito T (2009) Imaging improves diagnosis of dementia with lewy bodies. Psychiatry Investig 6: 233-240.

18. Tateno M, Kobayashi S, Shirasaka T, Furukawa Y, Fujii K, et al. (2008) Comparison of the usefulness of brain perfusion SPECT and MIBG myocardial scintigraphy for the diagnosis of dementia with Lewy bodies. Dement Geriatr Cogn Disord 26: 453-457.

19. Tateno M, Utsumi K, Kobayashi S, Takahashi A, Saitoh M, et al. (2008) Usefulness of a blood flow analyzing program 3DSRT to detect occipital hypoperfusion in dementia with Lewy bodies. Prog Neuropsychopharmacol Biol Psychiatry 32: 1206-1209.

20. Matsuda H (2007) [Neurological diseases and SPECT-analysis using easy Zscore imaging system (eZIS)]. Brain Nerve 59: 487-493.

21. Takeuchi R, Sengoku T, Matsumura K (2006) Usefulness of fully automated constant ROI analysis software for the brain: 3DSRT and FineSRT. Radiat Med 24: 538-544.

22. Kobayashi S, Tateno M, Morii H, Utsumi K, Saito T (2009) Decreased cardiac MIBG uptake, its correlation with clinical symptoms in dementia with Lewy bodies. Psychiatry Res 174: 76-80.

23. Hixson JE, Vernier DT (1990) Restriction isotyping of human apolipoprotein E by gene amplification and cleavage with HhaI. J Lipid Res 31: 545-548.

24. Ueki A, Kawano M, Namba Y, Kawakami M, Ikeda K (1993) A high frequency of apolipoprotein E4 isoprotein in Japanese patients with late-onset nonfamilial Alzheimer's disease. Neurosci Lett 163: 166-168.

25. Yamanaka H, Kamimura K, Tanahashi H, Takahashi K, Asada T, et al. (1998) Genetic risk factors in Japanese Alzheimer's disease patients: alpha1-ACT, VLDLR, and ApoE. Neurobiol Aging 19: S43-46.

26. Brecht WJ, Harris FM, Chang S, Tesseur I, Yu GQ et al. (2004) Neuronspecific apolipoprotein e4 proteolysis is associated with increased tau phosphorylation in brains of transgenic mice. J Neurosci 24: 2527-2534

27. Kawanishi C, Suzuki K, Odawara T, Iseki E, Onishi H, et al. (1996) Neuropathological evaluation and apolipoprotein $\mathrm{E}$ gene polymorphism analysis in diffuse Lewy body disease. J Neurol Sci 136: 140-142.
28. Okuizumi K, Onodera O, Tanaka H, Kobayashi H, Tsuji S, et al. (1994) ApoEepsilon 4 and early-onset Alzheimer's. Nat Genet 7: 10-11.

29. Kawamata J, Tanaka S, Shimohama S, Ueda K, Kimura J (1994) Apolipoprotein E polymorphism in Japanese patients with Alzheimer's disease or vascular dementia. J Neurol Neurosurg Psychiatry 57: 1414-1416.

30. Dai XY, Nanko S, Hattori M, Fukuda R, Nagata K, et al. (1994) Association of apolipoprotein E4 with sporadic Alzheimer's disease is more pronounced in early onset type. Neurosci Lett 175: 74-76.

31. Carrillo Garcia F, Gil Neciga E, Alberca R, Garcia-Solis D, Millan J, et al. (2008) [Apolipoprotein E4 in dementia with Lewy bodies]. Neurologia 23: $152-156$.

32. Engelborghs S, Dermaut B, Goeman J, Saerens J, Marien P, et al. (2003) Prospective Belgian study of neurodegenerative and vascular dementia: APOE genotype effects. J Neurol Neurosurg Psychiatry 74: 1148-1151.

33. Cummings JL (2000) Cognitive and behavioral heterogeneity in Alzheimer's disease: seeking the neurobiological basis. Neurobiol Aging 21: 845-861.

34. Jacobs D, Sano M, Marder K, Bell K, Bylsma F, et al. (1994) Age at onset of Alzheimer's disease: relation to pattern of cognitive dysfunction and rate of decline. Neurology 44: 1215-1220.

35. Fujimori M, Imamura T, Yamashita H, Hirono N, Ikejiri Y, et al. (1998) Age at onset and visuocognitive disturbances in Alzheimer disease. Alzheimer Dis Assoc Disord 12: 163-166.

36. Imamura T, Takatsuki Y, Fujimori M, Hirono N, Ikejiri Y, et al. (1998) Age at onset and language disturbances in Alzheimer's disease. Neuropsychologia 36: 945-949.

37. Lane R, He Y, Morris C, Leverenz JB, Emre M, et al. (2009) BuChE-K and APOE epsilon4 allele frequencies in Lewy body dementias, and influence of genotype and hyperhomocysteinemia on cognitive decline. Mov Disord 24: 392-400.

38. McKeith I, Fairbairn A, Perry R, Thompson P, Perry E (1992) Neuroleptic sensitivity in patients with senile dementia of Lewy body type. BMJ 305: 673-678.

39. Farrer LA, Cupples LA, Haines JL, Hyman B, Kukull WA, et al. (1997) Effects of age, sex, and ethnicity on the association between apolipoprotein $\mathrm{E}$ genotype and Alzheimer disease. A meta-analysis. APOE and Alzheimer Disease Meta Analysis Consortium. JAMA 278: 1349-1356.

40. Ishii K, Tamaoka A, Mizusawa H, Shoji S, Ohtake T, et al. (1997) Abeta1-40 but not Abeta 1-42 levels in cortex correlate with apolipoprotein E epsilon4 allele dosage in sporadic Alzheimer's disease. Brain Res 748: 250-252.

41. Kosaka K (1990) Diffuse Lewy body disease in Japan. J Neurol 237: 197-204. 